\title{
Automated image analysis for trajectory determination of single drop collisions
}

\author{
Johannes Kamp ${ }^{\mathrm{a}, *}$, Ronny Hänsch ${ }^{\mathrm{b}}$, Gregor Kendzierski ${ }^{\mathrm{a}}$, Matthias Kraume ${ }^{\mathrm{a}}$, Olaf Hellwich ${ }^{\mathrm{b}}$ \\ ${ }^{a}$ Chair of Chemical E Process Engineering, Technische Universität Berlin, FH 6-1, Fraunhoferstr. 33-36, 10587 Berlin, Germany \\ ${ }^{b}$ Computer Vision and Remote Sensing, Technische Universität Berlin, MAR 6-5, Marchstr. 23, 10587 Berlin, Germany
}

\begin{abstract}
The fundamental analysis of drop coalescence probability in liquid/liquid systems is necessary to reliably predict drop size distributions in technical applications. For this crucial investigation two colliding oil drops in continuous water phase were recorded with different high speed camera set-ups under varying conditions. In order to analyse the huge amount of recorded image sequences with varying resolutions and qualities, a robust automated image analysis was developed. This analysis is able to determine the trajectories of two colliding drops as well as the important events of drop detachment from cannulas and their collision. With this information the drop velocity in each sequence is calculated and mean values of multiple drop collisions are determined for serial examinations of single drop collisions. Using the developed automated image analysis for drop trajectory and velocity calculation, approximately 1-2 recorded high speed image sequences can be evaluated per minute.
\end{abstract}

Keywords: image analysis, drop collision, high speed imaging, collision velocity, trajectory

\section{Introduction}

The understanding and successful description of liquid/liquid dispersions is important for many technical applications. Process operations like e.g. mixing, extraction, or separation inherently comprise dispersions and also many products like e.g. milk or cosmetic creams and lotions consist of or contain emulsions which are stabilised liquid/liquid dispersions. The performance of the processes and the product quality are mainly determined by the drop size distribution of the dispersion. The droplets within the dispersion can either break due to turbulent eddies and shear stress or confluence after a collision of two droplets which is known as coalescence. The two phenomena drop breakage and coalescence determine the drop size distribution. State of the art modelling approaches describe the drop size distribution by separate kernels for breakage and coalescence rate. These so called population balance equations allow the separate implementation of

\footnotetext{
* corresponding author Kamp)

Email address: johannes.kamp@tu-berlin.de (Johannes

URL: www.vt.tu-berlin.de (Johannes Kamp),

www.cv.tu-berlin.de (Ronny Hänsch)
}

breakage and coalescence which occur simultaneously in a general dispersion $[1,2,3]$. Several research groups investigated the breakage $[4,5,6,7]$ and coalescence $[8,9,10,11]$ separately in order to validate and improve these modelling approaches. The investigations use digital high speed cameras to obtain a high spatial and temporal resolution. The use of high speed imaging is necessary because the interesting time scales lie in the order of microseconds and even below [12]. The analysis of the generated data is a crucial aspect especially for serial examinations which are necessary for randomly distributed events like coalescence. The huge amount of recorded image sequences has to be analysed automatically by applying specific computer vision methods.

This paper proposes a first step in the direction of fully automatic coalescence detection: Based on the test cell described in [8], a system is developed to automatically detect and track drops within the videos. From the established trajectories the time points of drop detachments as well as drop collision are reliably determined. This allows on the one hand to derive physical parameters (such as rise velocity) that are known to have a substantial influence on the coalescence probability and on the other hand restricts the amount of data in which coalescence might happen. 


\subsection{Coalescence in liquid/liquid systems}

During a collision of two droplets with a certain relative velocity, the droplets deform each other and a thin liquid film of continuous phase is enclosed between the opposing surfaces of the drops. This film is so thin that the drops seem to be in contact with each other in visual observations (see Figure 1 at $\mathrm{t}=0.00 \mathrm{~ms}$ ). The film drains during further interaction of the droplets and the distance between the interfaces diminishes. If the "contact" of the drops lasts long enough for the film to drain sufficiently, a certain critical distance between the droplet surfaces is reached at which the interfaces merge spontaneously (also referred to as critical film thickness). The moment and point at which this film rupture occurs is affected by natural fluctuations and makes the coalescence a stochastic process [13]. After the film rupture (see Figure 1 at $\mathrm{t}=4.71 \mathrm{~ms}$ ) a coalescence bridge is built (see Figure 1 at $\mathrm{t}=4.78 \mathrm{~ms}$ $6.00 \mathrm{~ms}$ ). The interfacial tension drives the confluence of the drops by contracting the mutual surface (see Figure 1 at $t>7.00 \mathrm{~ms}$ ). The steps of drop collision, deformation and film drainage are mechanistic and can be described by several models available in literature [2]. The stochastic phenomenon is the moment at which the draining film ruptures and coalescence occurs. Therefore, common modelling approaches describe the mechanistic parts and assume a mean critical film rupture thickness as coalescence criterion. Furthermore, the models account for the stochastic film rupture by predicting only coalescence probabilities within a droplet swarm and do not predict single coalescence events. So-called film drainage models regard the drainage and contact times as random variables and put them in relation to each other [2]. In the model representation of film drainage a repulsion of drops after collision can be explained as follows: if the drainage of the thin film takes longer than the contact time of the droplets they repulse each other elastically and do not coalesce. The velocity and the manner of film drainage depends on various influencing parameters i.e. physical properties of the phases (density, viscosity), interfacial tension, surface active components, dissolved ions, $\mathrm{pH}$ value, mass transfer, relative velocity, oscillations, drop sizes, and many more [14]. Even under apparent constant conditions coalescence itself is a stochastic event and the result of a single droplet collision (coalescence or repulsion) is not predictable. The drop shapes appearing in Figure 1 are representative for the coalescence events recorded. The drop sizes vary from $\mathrm{d}=1.5-3.0 \mathrm{~mm}$ in the experiments so that also unequally sized droplets collide with each other.

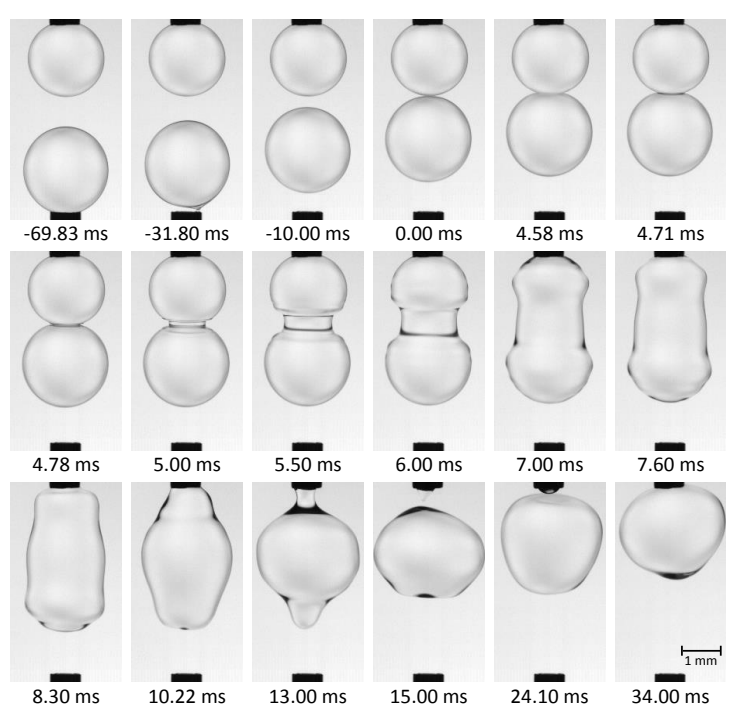

Figure 1: Image sequence of two coalescing drops $\left(d_{t o p}=2.0 \mathrm{~mm}\right.$, $d_{\text {bottom }}=2.2 \mathrm{~mm}$ ) recorded with VisionResearch Phantom v711 (camera system (3)) at 100,000 frames per second. The time of visual 'contact' is set to $t=0 \mathrm{~s}$. Full video sequence in supplementary material.

\subsection{Image analysis}

The current experimental set-up states several challenges that need to be handled by the data analysis modules.

1. It is not sufficient to merely detect the drop in the image, but its exact position as well as $2 \mathrm{D}$ radius has to be determined. The video-based estimation of the target variables (such as drop velocity, contact angle, etc.) requires a certain accuracy of the drop localisation. Any measurement error at this stage has a direct influence on the final results.

2. The images of the droplets are virtually untextured due to the transparency of the fluids. Only the light dispersion on the surface of the droplets causes weak intensity gradients in the images, which frequently diminish locally during drop movement. This already weak optical signal is further degraded by the high speed cameras. The extremely short exposure times of these cameras require a strong illumination. Even high power LEDs cannot guarantee a constant and homogeneous lighting during the whole image acquisition. Due to inhomogeneities in the LED fields or vignetting by the camera lenses, the images are degraded by global gradients in intensity as well as local image noise. The weak texture of the objects on the one hand 
and the relatively strong artefacts of the image acquisition process on the other hand require a robust image-based tracking system, which is able to resolve such issues.

3. Last but not least, the large amount of data is a challenge by itself. Although a high accuracy of the drop localisation under difficult image characteristics is required, it is not feasible to apply highly complex, time consuming methods. The data is analysed offline without any need for realtime processing. However, hundreds of video sequences (i.e. 3-8 gigabyte of data) per parameter set have to be analysed to obtain a sufficient sample size.

\section{Materials and Methods}

\subsection{Experimental set-up}

The coalescence investigations were performed in a test cell designed for the collision of a rising drop with a pendant drop fixed on a vertical cannula. The applied liquid phases were toluene as disperse and water as continuous phase according to the EFCE standard test systems for extraction [15]. The entire automated set-up was described in detail by Kamp and Kraume [8]. Using Hamilton syringe pumps the drop volumes could be determined precisely and drop sizes between $1.5 \mathrm{~mm}$ $3.0 \mathrm{~mm}$ were investigated. The relative collision velocity of the droplets was determined by varying the rising distance of the bottom drop, which was detached from a cannula at the bottom of the test cell. Therefore, the distance between the cannulas could be varied from drop contact up to $100 \mathrm{~mm}$ [8]. Three different monochrome digital high speed camera systems were used to record the drop collisions:

1. Photonfocus MV-D752-160-CL-8 (maximum resolution of $752 \times 582$ pixels at a frame rate of $350 \mathrm{fps}$ ) with frame grabber board Silicon Software microenable III, Pentax TV lens $12 \mathrm{~mm}$ and synchronised backlight LED flash CCS LDL-TP100/100-R,

2. Optronis CL600X2-M (maximum resolution of $1280 \times 1024$ pixels at a frame rate of $500 \mathrm{fps}$ ) with frame grabber board Silicon Software microenable IV, Pentax TV lens $12.5 \mathrm{~mm} \mathrm{1:1.4} \mathrm{and}$ synchronised backlight LED flash CCS LDL-TP100/100-R,

3. VisionResearch Phantom v711 monochrome (maximum resolution of $1280 \times 800$ pixels at a frame rate of $7530 \mathrm{fps}$ ) with $\begin{array}{llllll}\text { Sigma APO macro lens } & 180 & \mathrm{~mm} & \text { F2.8 }\end{array}$
EX DG OS HSM and continuous LED backlight GS Vitec MultiLED LT-V8-15.

The frame rate of all CMOS camera systems can be increased by decreasing the resolution of the recorded frame. Most of the image sequences were recorded sideways to achieve maximal spatial and temporal resolution with the high speed cameras. This is due to the design of the CMOS sensors and how the data is read from them: a reduction of lines leads to a higher maximal frame rate than reducing the same amount of rows which are read from the sensor. Therefore, a 90-degree rotation is beneficial for an area of interest with smaller width than height.

At higher frame rates the exposure time decreases and a bright illumination becomes more important. Thus, all illumination methods reach a certain limit at a certain high frame rate with corresponding short exposure time. In general, LED illumination is preferable against other methods because the heat radiation is much smaller.

To achieve a database of sufficient size for statistical analysis at least 100 droplet collisions were recorded with camera system (1) and (2). The number of necessary image sequences was determined by Kamp and Kraume [8] by analysing the trend of coalescence probability over the analysed sequences. The recording was implemented entirely in the LabVIEW automation of the test cell. In the case of camera system (3) the recording had to be triggered manually and, thus, fewer but higher resolved sequences were recorded. With these three cameras systems many image sequences were recorded with varying quality as well as temporal and spatial resolution. The applied image analysis has to cope with pictures of different orientation, resolution, frame rate, and illumination.

For each recorded image sequence a parameter file was created containing the known experimental conditions (drop diameters, frame rate, temperature, etc.). This information is used to enhance the image analysis.

As the coalescence probability is very sensitive against even small impurities, the purity of the system was checked before each experimental run. The rising path of droplets is analysed because most critical impurities alter the surface mobility and, therefore, the drag coefficient and the rising velocity. The drop rise velocity was determined as described in Villwock et al. [9] and compared to the theoretical trajectory of a drop with fully mobile interface $[16,17]$.

\subsection{Image analysis}

The image analysis is realized in Matlab using the integrated Image Analysis toolbox. The source code 
of the methods described in this work is provided in the supplementary material of the digital publication together with a link to the latest version of the code (see AppendixA.2)

\section{Automated image analysis}

Figure 2 illustrates the general workflow of the proposed framework to automatically analyse the acquired high speed image sequences. The framework is based on two principle ideas: First, to exploit the available prior knowledge (e.g. test cell layout, drop size, theoretic drop movement, etc.) as much as possible. Second, moving from easy to complicated tasks by using already acquired knowledge, instead of solving the hard task of drop detection directly. Each of the individual modules is described in detail in the following subsections. After loading the video data and the corresponding parameter file, which summarizes the experimental set-up, the individual images are preprocessed to ease the subsequent analysis tasks. The orientation of the image sequence is automatically determined based on the knowledge that the droplets move only in one direction (i.e. upwards). In a next step the cannulas are located. Based on the position and shape of the cannula, the centre points of the two drops within the first frames are predicted. In subsequent frames the drop positions are first predicted based on a simple motion model and then refined based on the image data of the current frame. As soon as the trajectory of the droplet is known, the events of drop detachment and collision are determined. Based on these two time stamps, different sequences of the same experimental set-up are aligned and averaged to enable a statistical interpretation of the acquired data.

The coordinate system was defined as follows: the point of origin lays in the upper left corner of the rotated image, $x$-coordinate increases rightwards and $y$ coordinate increases downwards.

\subsection{Preprocessing}

As described in Section 2.1, the images of the test cell are captured by a high-speed camera, which provides an image sequence $\left\{I_{t}\right\}_{t=1, \ldots, N}$ of $N$ images with a given frame rate. The images $I_{t}$ (Figure $3 \mathrm{a}$ shows an example) are degraded by global gradients in intensity as well as local image noise. Both effects need to be reduced before any subsequent image analysis task.

The normalization of inhomogeneous lighting is strongly connected with the similar computer vision task of colour normalization (see for example [18]). One simple yet successful approach is the computation

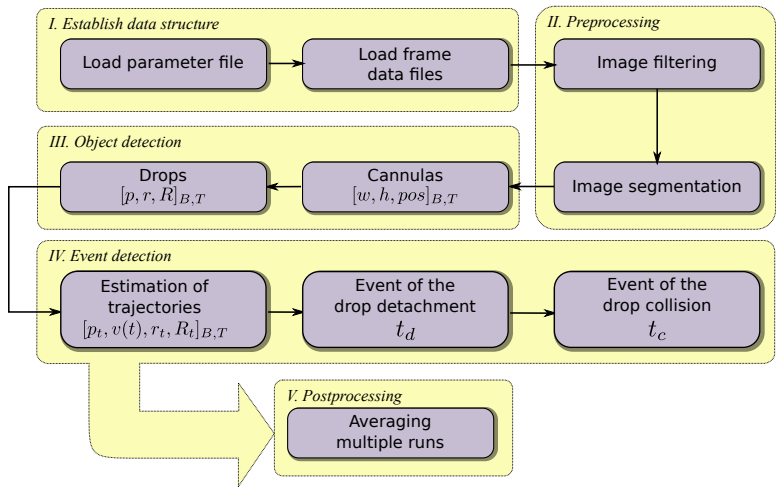

Figure 2: Workflow of the proposed method

of the Self Quotient Image (SQI, [19, 20]), which is a fast method to minimize the effect of inhomogeneous lighting conditions on images. The SQI $Q_{t}$ of the image $I_{t}$ at time $t$ is defined by Eq. 1 , where $\hat{I}_{t}=G * I_{t}$ is the input image smoothed by a Gaussian filter $G$.

$$
Q_{t}(x, y)=\frac{I_{t}(x, y)}{\hat{I}_{t}(x, y)}
$$

The standard deviation of the filter is defined empirically and is set to 10 pixel for all experiments.

Since $I_{t}(x, y) \approx \hat{I}_{t}(x, y)$ over (locally) homogeneous regions, $Q_{t}(x, y)$ is close to one independent from the absolute value of the intensity within these regions, while the texture in inhomogeneous regions is preserved (see Figure 3b). The halo-effect around object borders is caused by the isotropic smoothing of the Gaussian filter. Although it can be prevented by edge-preserving smoothing operators (as for example the bilateral filter [21]), it does not have a negative effect on the subsequent analysis in this work. The Gaussian filter proved sufficient and is mainly chosen for the sake of a small computational load.

Since global image gradients have been removed within the SQI, only local image gradients remain. Those are mainly caused by the objects of interests, which are either blocking (cannulas) or dispersing (droplet borders) the light rays. Both objects are generally darker than the background and can be detected by a simple threshold operation (Eq. 2), where $\theta$ is a threshold which is automatically set to the average intensity of $Q_{t}$ in all experiments. The effect of the thresholding is shown in Figure 3c.

$$
T_{t}(x, y)=\left\{\begin{array}{cc}
1 & \text { if } Q_{t}(x, y)<\theta \\
0 & \text { otherwise }
\end{array}\right.
$$




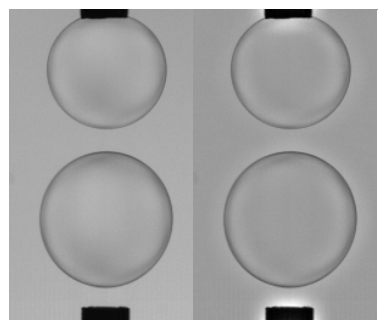

(a) Original input image $I_{t}$

(b) SQI $Q_{t}$

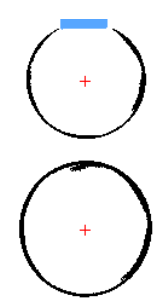

Centre $\hat{p}_{t}$ estimation
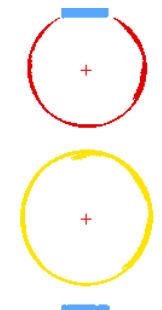

(f) Border pixel $O_{t}$ detection

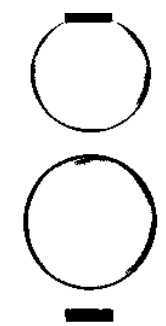
image $T_{t}$
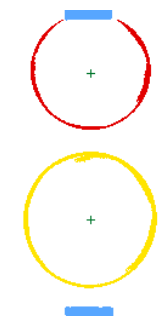

(g) Centre $p_{t}$ calculation (c) Thresholded

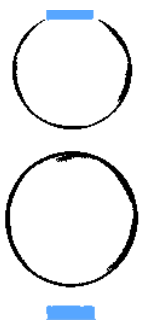

(d) Cannula detection
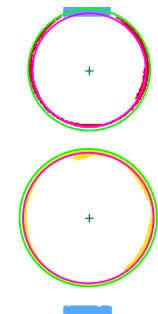

(h) Mean radi calculation
Figure 3: Intermediate results of image processing steps

\subsection{Image orientation detection}

In a first step, the orientation of the images is automatically determined by integrating the image intensity over each image dimension. The distribution of intensity values stays nearly constant over time if integrated parallel to the drop movement, while it varies with the drop movement if integrated perpendicularly. The vertical image direction is determined as the direction in which the intensity distribution varies most over time. The direction of this change defines which of the two horizontal image borders shows the lower and upper cannula. If necessary, the images are rotated such that the lower cannula is located at the lower border of the image and the upper cannula at the upper image border. After the orientation of the image is known, the objects, namely cannulas (see Section 3.3.1) and drops (see Section 3.3.2), are detected.

\subsection{Object detection}

\subsubsection{Cannula detection}

The cannulas are of no direct interest. Nevertheless, precise knowledge of their location and dimension is crucial for the following reasons:

1. They can be detected much easier than the drops. Their position is (at least coarsely) known a priori since the image orientation is already known and the orientation of the cannulas does not change within the current experimental set-up. The image of the cannulas stays constant and is a very dominant image feature, while the droplets strongly change in appearance during rise, contact, and coalescence/repulsion.

2. At the beginning of the sequence, both drops are connected to the cannulas. Knowing the shape and position of the cannula provides a solid initialization to find the position of the droplets within the first frame.

3. The physical size of the cannulas is known, while the size within the image is derived from analysing their detected shape. The relation of physical and imaged size of the cannulas provides the necessary information to compute the expected drop radius in pixels as well as the theoretical drop velocity in pixels per second.

The detection of the cannulas is based on the knowledge that they are represented by large dark objects, which are connected to the image borders and stay constant over the whole image sequence. In a first step a cumulated image $\bar{T}$ is generated according to Equation 3 by summing over the whole preprocessed image sequence, where $T_{t}$ is the thresholded image of time $t$ (see Equation 2).

$$
\bar{T}=\sum_{t=1}^{N} T_{t}
$$

The application of the above constraints to $\bar{T}$ leads to the rejection of all other objects within the image besides the two cannulas. The drop on top changes only after contact with the rising drop and stays constant otherwise. This can cause small artefacts in the form of drop pixels that remain in the cumulated image. In order to delete these erroneous pixels, the shape of each cannula is refined in a second step. The cannula is assumed to be coarsely aligned with the image axes. The histogram of cannula pixels cumulated horizontally and vertically resembles a piecewise constant function as shown in Figure 4. Width and height of the cannula are determined as the width of the major part of the histogram. The exact shape is defined by the extremal pixel positions of the four quadrants around the centre of gravity.

\subsubsection{Drop detection}

The expected drop radius in millimetre as well as the physical size of the cannula are known from the corresponding parameter file. Image orientation as well as cannula shape and position are estimated from the given 


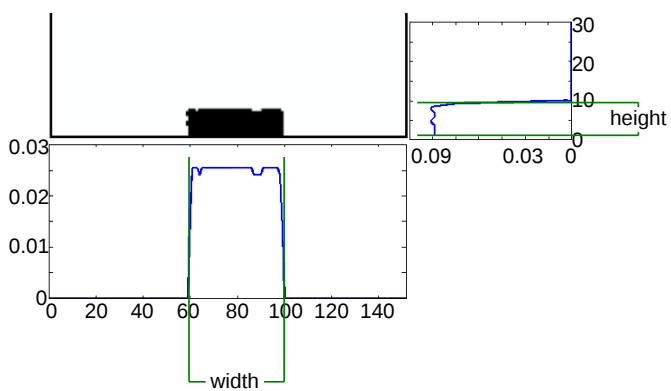

Figure 4: Refinement of cannula shape

image data. The centres of the upper (lower) cannula borders $\left.c_{\text {top }}^{u}\left(c_{\text {top }}^{l}\right)\right)$ are calculated from the detected cannula pixels. By relating physical and imaged size of the cannula, all metric length information can be transformed into pixel measurements (and vice versa).

In the following the object detection is only described for the rising drop. The detection of the fixed droplet on top is done accordingly.

Based on the expected drop radius $\hat{r}$ in pixel, the expected centre of the bottom drop $\hat{p}_{t}$ within the first frame $(t=0)$ can easily be determined as $\hat{p}_{0}=c_{\text {top }}-\hat{r}$. This predicted drop centre is quite accurate, since no motion took place yet and the test cell is able to accurately generate drops of a specific size.

For the following frames a simple motion model could be used to predict the drop centre $\hat{p}_{t_{i+1}}$ in the next frame $i+1$ based on the drop centre $\hat{p}_{t_{i}}$ in the $i$-th frame. However, small differences between real and theoretical drop movement would accumulate over time, causing the predicted drop centre to drift away from the real drop centre. That is why the predicted drop centre $\hat{p}_{t}$ needs to be adjusted according to the image data at this time step leading to a refined estimated drop centre $p_{t}$.

All foreground pixels within a circle of $1.5 \cdot \hat{r}$ radius around the expected drop centre $\hat{p}_{t}$ are selected, i.e. $B_{t}=\left\{(x, y) \mid T_{t}(x, y)=1,\left\|(x, y)-\hat{p}_{t}\right\|<1.5 \hat{r}\right\}$. All pixels within $B_{t}$ lie close to the drop boundary, since the interior of the drop is transparent and only the border of the drop appears dark due to light dispersion. However, due to vanishing gradients and the segmentation with a fixed threshold, it is unlikely that the pixels in $B_{t}$ form a one-pixel wide, connected curve. In order to refine the border of the drop, the area around $\hat{p}_{t}$ is divided into angular intervals $\Delta \theta$, defined by Equation 4 using the mean radius (see Equation 6) of the previous frame $\bar{r}_{t-1}$ and a border section of $b=2 \mathrm{px}$. The upper left corner of Figure 5 shows a few exemplary intervals $\Delta \theta$.

$$
\Delta \theta=\arccos \left(1-\frac{b^{2}}{2 \bar{r}_{t-1}^{2}}\right)
$$

The pixel in $B_{t}$ that has the maximal distance from $\hat{p}_{t}$ within $\Delta \theta$ is considered as part of the outer border pixels $O_{t}$. Figure 5 shows the resulting outer drop border pixels $O_{t}$ marked as black squares and the remaining drop border pixels $B_{t} \backslash O_{t}$ as grey squares.

A simple computation of the centre of gravity of $O_{t}$ would shift $p_{t}$ to the side of the drop on which more border pixels are detected. Instead, the refined drop centre $p_{t}$ is determined by computing a weighted average of the outer border pixels $o_{n} \in O_{t}\left(n=1, \ldots, N:=\left|O_{t}\right|\right)$ where the weight depends on the angle to their next neighbours (see Figure 5):

$$
p_{t}=\frac{1}{4 \pi} \sum_{n=1}^{N}\left(\theta_{n-1}+\theta_{n+1}\right) \cdot o_{n}
$$

where $o_{0}:=o_{N}$ and $o_{N+1}:=o_{1}$.

Using this method the real drop centre can be calculated even if the border is undisclosed and unequally thick.

The minimal and maximal distance between the computed centre $p_{t}$ and the outer border pixels $O_{t}$ determine the radii $r_{t}, R_{t}$ of the minimal and maximal outer boundary, respectively, which are depicted as magenta and green circles in Figure 5. The mean radius $\bar{r}_{t}$ is computed according to Equation 6.

$$
\bar{r}_{t}=\frac{r_{t}+R_{t}}{2}
$$

When the drop centre $p_{t}$ in frame $t$ is known, the expected drop centre $\hat{p}_{t+1}$ in frame $t+1$ is estimated as $\hat{p}_{t+1}=p_{t}+f_{t}$, where $f_{t}$ is calculated from the drop velocity known from the previous frames: $f_{t}=v_{t} \cdot \Delta t=\left(p_{t}-p_{t-1}\right)$. Alternatively, a motion model e.g. $f_{t}=a / 2 t^{2}$ for linear accelerated motion could be used. The new expected drop centre is refined by the same procedure as discussed above.

\subsection{Event detection}

Although the detachment of the bottom drop from the lower cannula is triggered by the system, the exact starting time of the drop movement is not known. The image recording is not synchronized with the movement of the droplet. It is only ensured, that the start of the movement as well as the drop collision is within the recorded time frame. Both events need to be detected automatically in order to derive robust and precise information about the drop movement. 


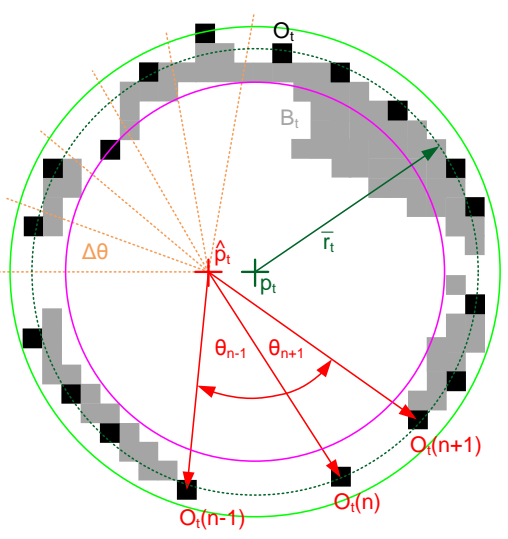

Figure 5: Maximal drop border pixels $O_{t}$ (black squares) are selected within intervals $\Delta \theta$ from the drop border pixels $B_{t}$ (grey squares). Minimal (magenta circle) and maximal (green circle) outer circles are determined from the extrema of $O_{t}$. Subsequently, the drop centre $p_{t}$ is determined from neighbouring pixels in $O_{t}$ (red labelling).

After the initial detection of the droplet in the first frame, its border is described by two circles with minimal and maximal outer radius around a common centre (see Section 3.3) which are depicted in magenta and light green in Figure $3 \mathrm{~h}$ and Figure 5. These two circles are used to mark a time interval during which drop and cannula separate. The beginning of this interval is defined as the moment, when the smallest distance between the pixels of the cannula to the inner ring is larger than one pixel. The time interval ends, when the smallest distance between the outer ring and the cannula is larger than one pixel. A second time interval is defined for the contact of the two droplets in a very similar way: The interval starts, when the outer circles of the drops are closer to each other than one pixel and it ends as soon as the inner circles touch each other.

Both intervals of the event detection are subsequently refined. Within the interval of the cannula detachment event of the bottom drop, the following procedure is carried out for each frame: The distances between the bottom drop border pixels and the upper border of the cannula are calculated (see Figure 6). A drop border pixel in $O_{t}$ is only considered if it lies within an angle of $\pm \theta=\frac{\pi}{4}$ from the vertical line of the drop's centre. For each side of this vertical line, the pixel with the smallest distance is selected and its distance to the drop's centre is projected onto the vertical line. The frame in which the mean value of these two distances is larger than 1.5 px marks the detachment event. The collision event between the two drops is refined within the found interval likewise by using the border pixels of the two drops.

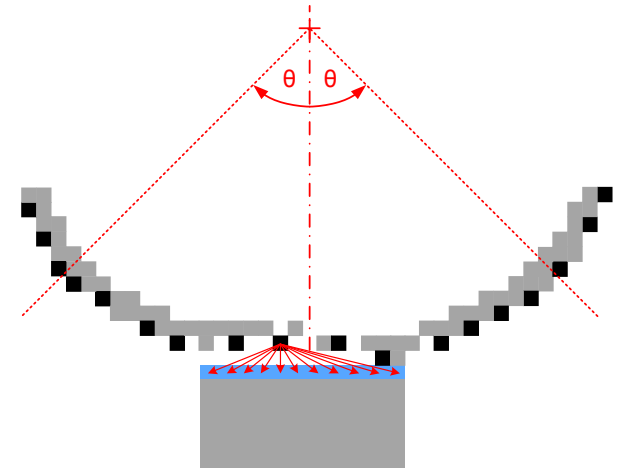

Figure 6: Refined cannula detachment detection visualising the distance calculation for one pixel schematically.

\subsection{Post-processing}

Once the coordinates of the drop centres are known, the estimation of the whole trajectory and the rise velocity is straightforward. The drop trajectory is defined as linear interpolation of the determined drop centres $p_{t}$ smoothed by a moving average over \pm 10 frames. Drop centre displacements greater than 2 px per frame are neglected since that would correspond to implausible accelerations. The resulting trajectory $\left(\bar{p}_{t}\right)$ of the rising droplet is used to calculate the drop velocity as the derivative of the trajectory.

The conversion factor from pixels to millimetres $c_{m m / p x}$ was determined during the cannula detection and the recorded frame rate fps is known from the parameter file of the experimental sequence. The trajectory in physical coordinates (millimetres and time) is calculated directly between the events of drop detachment and collision. The drop velocity in pixels per frame is calculated as temporal derivative of the drop centres by the central differential quotient:

$$
v(t)=\frac{\bar{p}_{t+1}-\bar{p}_{t-1}}{2}
$$

For experimental analysis the vertical component of the rise velocity versus the drop's rise height $v_{x}\left(x_{t}\right)$ is important. These values are known but might contain multiple function values of the velocity at identical rise heights (i.e. $x_{t_{i}}=x_{t_{j}}$ for $i \neq j$ ) due to oscillating droplets or fluctuations in the centre determination. Therefore, $x_{t}$ is converted to a strictly increasing sequence $x_{\bar{t}}$ by rejecting frames $t_{i}$ for which $x_{t_{i}}=x_{t_{j}}$ with $j<i$. The drop rise velocity is then only plotted against the strictly increasing height, i.e. $v_{x}\left(x_{\bar{t}}\right)$.

From all sequences with identical experimental parameters the mean values $\bar{v}_{x}(t)$ and $\bar{v}_{x}\left(x_{\bar{t}}\right)$ as well as the 
maximal, minimal, and standard deviation values are derived. Unevenly distributed velocity values are interpolated linearly to equally sized intervals of $t$ and $\bar{x}$ for each sequence.

\section{Results and Discussion}

In order to demonstrate the output of the image analysis, the single sequence recorded with camera system (3) and shown above in Figures 1 and 3 was analysed exemplary. The initial distance between the two drops $\left(d_{\text {top }}=2.0 \mathrm{~mm}, d_{\text {bottom }}=2.2 \mathrm{~mm}\right)$ was $0.8 \mathrm{~mm}$ or $38 \mathrm{px}$. The results are plotted in Figure 7 . The trajectory (in this case the rising height $x_{\bar{t}}$, where $x_{0}:=0$ ) of the rising drop centre point $\bar{p}_{t}$ is shown over the frame number. The first detected centre point was defined as zero in this representation. In the same graph the calculated velocity is shown together with the detected events of drop detachment from the bottom cannula and drop collision. It can be seen Figure 7, that the centre point does not rise with constant acceleration but fluctuates especially in the region of frame number 3800 . Analysing the original sequence shows that the bottom drop does not instantly detach from the cannula entirely but a thin liquid bridge keeps the droplet connected to the cannula. The detachment, however, was already detected at frame 1889. The liquid bridge is present until frame 3816 after which the position of the detected centre increases rapidly. For later analysis this deviation is negligible because the velocity over height is more important than over time and the difference of the centre point between the two detachment points is only 2 pixels.

Although the final trajectory $\bar{p}_{t}$ does not contain any strong changes in drop positions, even slight fluctuations of the determined centres are amplified in the calculated velocity (green curve in Figure 7). Additionally, the calculated velocity of the non-smoothed centre points $p_{t}$ is shown in Figure 7 as light green crosses. These non-smoothed velocities show significant fluctuations (scattering up to $\pm 0.2 \mathrm{px} /$ frame, exceeding the displayed scale) because already a small centre point shift causes a high velocity at these high frame rates. In the shown sequence with a recorded frame rate of $100,000 \mathrm{fps}$ and a conversion factor of $c_{m m / p x}=0.021 \mathrm{~mm} / \mathrm{px}$ a jump of 1 pixel per frame would result in a velocity of above $2000 \mathrm{~mm} / \mathrm{s}$ whereas the actual velocity of the rising drop is two orders of magnitude smaller. This effect can not be avoided due to the discrete form of the digital image and the limitations of digital resolution.

Applying the above described moving average to the drop centre trajectory, the determined velocity shows

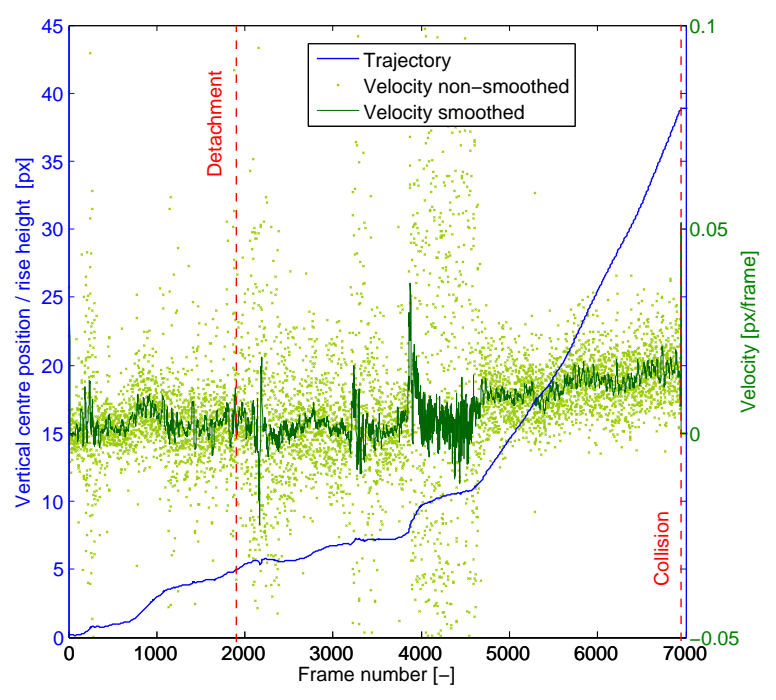

Figure 7: Trajectory of single sequence used for visualisations in this work together with the calculated velocity using the determined trajectory and using a smoothed trajectory by applying a moving average

less fluctuations (green curve in Figure 7). This illustrates the necessity of the smoothing by a moving average in the trajectory determination. The fluctuations can be assumed to have zero mean value. Averaging reduces the variance of this noise and leads to an velocity estimate, which is closer to the true velocity than the original calculations. Thus, a broader smoothing interval might be more suitable at these high frame rates.

In the shown sequence the experimentally adjusted drop distance of 38 px was detected successfully by the automated image analysis which computed a collision point after rise height of 38.8 px (see Figure 7).

The mean value calculation is shown exemplarily for 98 image sequences recorded with high speed camera system (1) at a frame rate of $1162 \mathrm{fps,} \mathrm{drop} \mathrm{sizes} \mathrm{of}$ $d_{\text {top }}=2.6 \mathrm{~mm}, d_{\text {bottom }}=2.5 \mathrm{~mm}$ and a drop distance of $4.3 \mathrm{~mm}$ or $98 \mathrm{px}$. The use of images from high speed camera system (1) shows the capability of the image analysis for different image resolution and quality. The selected set of image sequences is representative for serial examinations of coalescence probability (see $[8,9])$. In Figure 8 the mean drop rise velocity is plotted versus the height of the drop rise including the minimal and maximal velocity values at the respective heights. Additionally, the mean value of the collision points is shown. There are no high-frequency fluctuations of the velocity visible as in Figure 7 because the same smoothing interval was used at much lower frame rate. Nevertheless, the resulting mean value calculation of a series of 


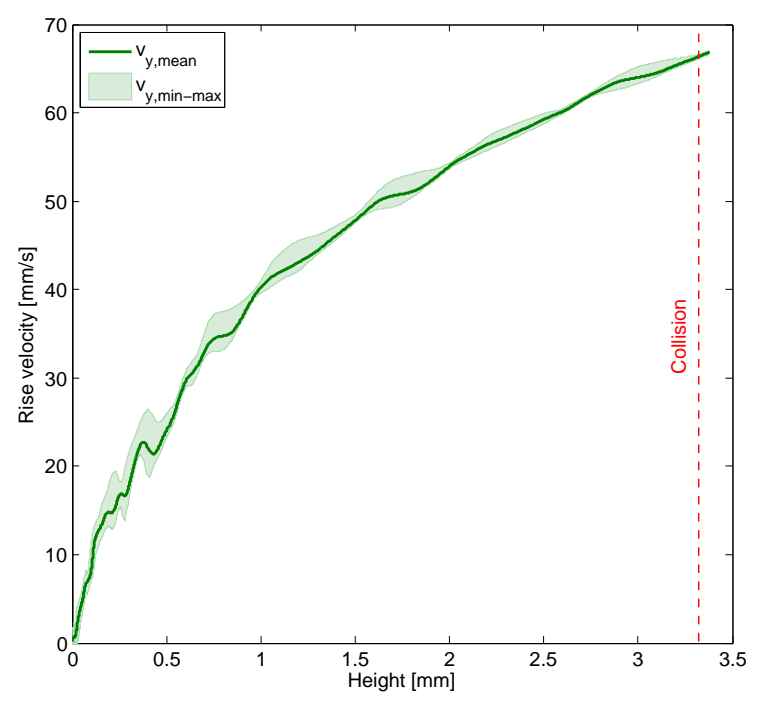

Figure 8: Mean velocity of 98 sequences including minimal and maximal values

drop trajectories like the one shown in Figure 7 would be similar to Figure 8 . The mean rise velocity shows fluctuations after detachment at a rise height of around $0.5 \mathrm{~mm}$ which is caused by slight variations of the drop detachment from the lower cannula. In general, the differences between the minimal and maximal velocities are remarkably small in all 98 sequences which emphasizes the good reproducibility of the experimental setup. The slight variations of the mean velocity during drop rise can be explained by oscillations of the drop induced by the detachment. The oscillation of a droplet affects its rise velocity directly due to the changing drag coefficient. Being able to evaluate the oscillation is an important outcome because it is known that the oscillation has a significant impact on the coalescence probability [22].

The computational performance of the image analysis is satisfactory. The evaluation of a single image sequence needs less than a minute on a single CPU with $2.66 \mathrm{GHz}$. The analysis of the 98 sequences (3.1 GB of data) shown above took about one hour. By parallelising the evaluation of the image sequences the computation time was reduced to 13 minutes using four CPU cores with $2.66 \mathrm{GHz}$.

\section{Summary and outlook}

A robust image analysis was developed to determine the trajectories of two colliding drops recorded by dif- ferent high speed cameras with varying temporal and spatial resolution and image quality.

The Self Quotient Image (SQI) filter was applied before thresholding to remove global image gradients and enhance the object detection. During the stepwise detection of the objects (cannulas and droplets) known information from the picture and experimental parameters are used effectively. This allows a fast and robust object detection. The cannulas, drop centre positions, and the events of drop detachment from the bottom cannula as well as drop collision are detected successfully. During post-processing the rise velocity of the bottom drop is calculated. Additionally, the mean values for a series of drop collisions can be determined which are necessary for serial examinations of single drop collision investigations.

The determined information from this image analysis allows the fundamental analysis of coalescence using high speed imaging. For the first time, the influence of collision velocity on coalescence probability can be evaluated based on the gained data. The findings can be used to validate existing coalescence models and develop new modelling approaches if necessary. With reliable and comprehensive models it is possible to predict the drop size distributions in liquid/liquid systems. This would allow a validation in lab scale apparatuses instead of excessive and expensive experimental investigations at different scales of process development in future. Thus, those comprehensive models could be used for process control and plant design of large scale applications.

Future development of the image analysis will evaluate the drop shape oscillations during rise and the detection of coalescence or repulsion events after drop collision. This information is important to further investigate fundamental influencing factors on coalescence probability.

\subsection{Acknowledgements}

The authors kindly thank the student worker Dorothea Löffler who developed the first routines for this image analysis. Financial support provided by the German Research Foundation (DFG) within the project KR 1639/19-2 is gratefully acknowledged.

\section{AppendixA. Supplementary material}

\section{AppendixA.1. Image processing as video sequence}

For the image sequence used in this work (see Figures 1, 3 and 7) a video sequence of the intermediate results of the image processing steps is provided (see 
Figure A.9). From left to right, it shows the original image sequence, the applied Self Quotient Image filter, the thresholded binary image, and the detected objects. The calculated drop centres are shown as white crosses, the minimal and maximal outer borders are depicted as magenta and green circles. The trajectory of the rising droplet is marked by red dots. The image sequence was recorded with camera system (3) at 100,000 fps. The video is played back with $100 \mathrm{fps}$ which results in a slow motion factor of 1000 .
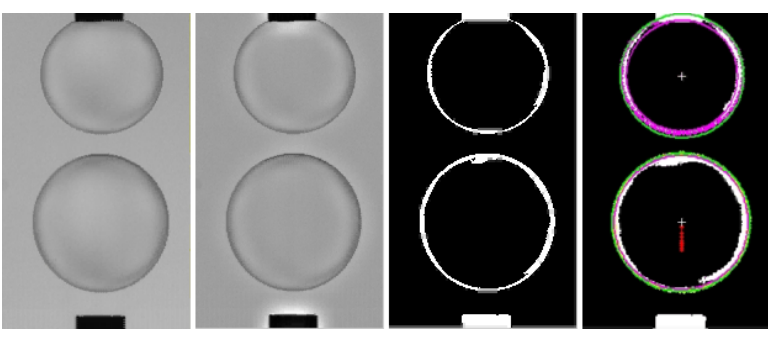

Figure A.9: Video of intermediate results of the image processing steps (original, SQI filtered $Q_{t}$, thresholded $T_{t}$ image and detected objects).

\section{AppendixA.2. Source code}

The source code of the methods described in this work is provided in the supplementary material of this digital publication under BSD 2-clause license. For trial runs a single image sequence recorded with camera setup (1) is provided. Additionally, the single image sequence used for visualisation in this work (camera system (3) with high frame rate of 100,000 fps) is available.

The source code can also be found on:

\section{- https://github.com/TU-Berlin-FGVT}

- http://www.rhaensch.de/droptrack.html

[1] Y. Liao, D. Lucas, A literature review of theoretical models for drop and bubble breakup in turbulent dispersions, Chemical Engineering Science 64 (15) (2009) 3389 - 3406. doi:10.1016/j.ces.2009.04.026.

[2] Y. Liao, D. Lucas, A literature review on mechanisms and models for the coalescence process of fluid particles, Chemical Engineering Science 65 (10) (2010) 2851-2864. doi:10.1016/j.ces.2010.02.020.

[3] D. Ramkrishna, Population Balances: Theory and Applications to Particulate Systems in Engineering, Academic Press, San Diego, 2000. doi:10.1016/B978-012576970-9/50001-1.

[4] S. Maaß, M. Kraume, Determination of breakage rates using single drop experiments, Chemical Engineering Science 70 (2012) 146-164. doi:10.1016/j.ces.2011.08.027.

[5] J. Solsvik, H. A. Jakobsen, Single drop breakup experiments in stirred liquid-liquid tank, Chemical Engineering Science 131 (2015) 219-234. doi:10.1016/j.ces.2015.03.059.
[6] J. Solsvik, H. A. Jakobsen, Single air bubble breakup experiments in stirred water tank, International Journal of Chemical Reactor Engineering 13 (4) (2015) 477-491. doi:10.1515/ijcre2014-0154.

[7] S. Nachtigall, D. Zedel, M. Kraume, Analysis of drop deformation dynamics in turbulent flow, Chinese Journal of Chemical Engineeringdoi:10.1016/j.cjche.2015.06.003.

[8] J. Kamp, M. Kraume, Influence of drop size and superimposed mass transfer on coalescence in liquid/liquid dispersions - test cell design for single drop investigations, Chem. Eng. Res. Des. 92 (4) (2014) 635-643. doi:10.1016/j.cherd.2013.12.023.

[9] J. Villwock, F. Gebauer, J. Kamp, H.-J. Bart, M. Kraume, Systematic analysis of single droplet coalescence, Chem. Eng. Technol. 37 (7) (2014) 1103-1111. doi:10.1002/ceat.201400180.

[10] R. T. Eiswirth, H.-J. Bart, A. A. Ganguli, E. Y. Kenig, Experimental and numerical investigation of binary coalescence: Liquid bridge building and internal flow fields, Physics of Fluids 24 (6) (2012) 062108. doi:10.1063/1.4729791.

[11] N. Kopriwa, Quantitative beschreibung von koaleszenzvorgängen in extraktionskolonnen, Ph.D. thesis, RWTH Aachen (2014).

[12] S. Thoroddsen, T. Etoh, K. Takehara, High-speed imaging of drops and bubbles, Annual Review of Fluid Mechanics 40 (1) (2008) 257-285. doi:10.1146/annurev.fluid.40.111406.102215.

[13] B. Radoev, A. Scheludko, E. Manev, Critical thickness of thin liquid films: theory and experiment., Journal of Colloid and Interface Science 95 (1983) 254-65. doi:10.1016/00219797(83)90094-2.

[14] G. V. Jeffreys, G. A. Davies, Coalescence of liquid droplets and liquid dispersions, in: Hanson [14], Ch. 14, pp. 495-584. doi:10.1016/B978-0-08-015682-8.50018-3.

[15] T. Misek, R. Berger, J. Schröter, Standard test systems for liquid extraction, 2nd Edition, Vol. 46 of EFCE Publication Series, The Institution of Chemical Engineers, Rugby, UK, Rugby, UK, 1985 .

URL http: //www . processnet .org/en/Literature.html

[16] Z.-G. Feng, E. E. Michaelides, Drag coefficients of viscous spheres at intermediate and high reynolds numbers, Journal of Fluids Engineering 123 (4) (2001) 841-849. doi: $10.1115 / 1.1412458$.

[17] M. Wegener, N. Paul, M. Kraume, Fluid dynamics and mass transfer at single droplets in liquid/liquid systems, International Journal of Heat and Mass Transfer 71 (2014) 475-495. doi:10.1016/j.ijheatmasstransfer.2013.12.024.

[18] J. Chang, R. Cabezas, I. Fisher, JohnW., Bayesian nonparametric intrinsic image decomposition, in: D. Fleet, T. Pajdla, B. Schiele, T. Tuytelaars (Eds.), Computer Vision - ECCV 2014, Vol. 8692 of Lecture Notes in Computer Science, Springer International Publishing, 2014, pp. 704-719.

[19] H. Wang, S. Li, Y. Wang, J. Zhang, Self quotient image for face recognition, in: Image Processing, 2004. ICIP '04. 2004 International Conference on, Vol. 2, 2004, pp. 1397-1400.

[20] A. Shashua, T. Riklin-raviv, The quotient image: Class-based re-rendering and recognition with varying illuminations, IEEE Trans. on Pattern Analysis and Machine Intelligence 23 (2001) 129-139. doi:10.1109/34.908964.

[21] C. Tomasi, R. Manduchi, Bilateral filtering for gray and color images, in: Computer Vision, 1998. Sixth International Conference on, 1998, pp. 839-846.

[22] G. F. Scheele, D. E. Leng, An experimental study of factors which promote coalescence of two colliding drops suspended in water - i, Chemical Engineering Science 26 (1971) 1867-1879. doi:10.1016/0009-2509(71)86030-X. 